Recepción: 25 / 03 / 2018

Aceptación: 04 / 05 / 2018

\title{
EI trabajo de campo como estrategia didáctica para el aprendizaje de estudiantes de trabajo social
}

Fieldwork strategy teaching for students of social work learning

Trabalho de campo como estratégia de ensino para a aprendizagem de alunos de trabalho social

Melida A. Barreto-Pico ${ }^{\mathrm{I}}$ asunción.dominio2015@outlook.es

\section{Correspondencia: asunción.dominio2015@outlook.es}

I Magister en Gerencia Educativa, Especialista en Diseño Curricular por Competencia, Trabajadora Social, Licenciada en Trabajo Social, Trabajo Social, Docente en la Universidad Laica Eloy Alfaro de Manabí, Manta, Ecuador. 


\section{Resumen}

Las estrategias de campo se constituyen en un medio didáctico que permite el contacto directo de los estudiantes con la realidad estudiada como medio de aprendizaje mucho más dinámico y vivencial, que rompen con la monotonía de los conocimientos recibidos en un aula de clases, donde los alumnos son capaces de confrontar la teoría con la práctica Este planteamiento permitió analizar el trabajo de campo como una estrategia didáctica para el aprendizaje de estudiantes de trabajo social. Metodológicamente el estudio fue de tipo descriptivo con diseño de campo y no experimental; la investigación se realizó con estudiantes de Trabajo social de la ULEAM, en la ciudad de Manta, durante el primer semestre del año 2017. La información se obtuvo a través de un instrumento tipo cuestionario y la técnica fue la encuesta. Los datos se analizaron desde la estadística descriptiva en la que se evidencio que el $80 \%$ de los alumnos manifestaron un aprendizaje actitudinal altamente favorable durante el proceso de enseñanza aprendizaje, en la que se concluyó que el trabajo de campo es una estrategia didáctica que en los estudiantes permite facilitar el proceso de enseñanza aprendizaje en estudiantes de la carrera de trabajo social.

Palabras clave: trabajo de campo; aprendizaje; estrategia didáctica y trabajo social.

\section{Abstract}

Field strategies are in an educational environment which allows students direct contact with reality studied as a means of learning much more dynamic and experiential, that break the monotony of the knowledge received in a classroom of classes, where students are able to confront theory with practice this approach allowed to analyze the field work as a teaching strategy for the learning of students of social work. Methodologically the study was descriptive with field and non-experimental design; the research was conducted with students of social work of the ULEAM, in the city of Manta, in the first half of the year 2017. The information was obtained through a questionnaire type instrument and technique was the survey. Data were analyzed from the descriptive statistics that are evidence that $80 \%$ of the students expressed a learning highly favorable attitude during the process of teaching and learning, in which it was concluded that field work is one teaching strategy that facilitates the process of teaching learning in students of social work students. 
Keywords: field work; learning; teaching strategy and social work.

\section{Resumo}

As estratégias de campo constituem uma ferramenta de ensino que permite contacto directo dos alunos com a realidade estudada como um meio de aprendizagem mais dinâmico e experiencial, que quebrar a monotonia do conhecimento recebido em uma sala de aula onde os alunos eles são capazes de confrontar a teoria com a prática. Esta abordagem permitiu analisar o trabalho de campo como uma estratégia didática para a aprendizagem de alunos do serviço social. Metodologicamente, o estudo foi descritivo com delineamento de campo e não experimental; A pesquisa foi realizada com estudantes de Serviço Social ULEAM na cidade de Manta, no primeiro semestre de 2017. As informações foram obtidas através de um instrumento tipo de questionário e a técnica foi a pesquisa. Os dados foram analisados a partir das estatísticas descritivas em que foi mostrado que $80 \%$ dos estudantes expressaram um aprendizado de atitudes muito favorável durante o processo de ensino-aprendizagem na qual concluiu-se que o trabalho de campo é uma estratégia de ensino na Os alunos podem facilitar o processo de ensinoaprendizagem em estudantes de carreira social.

Palavras chave: trabalho de campo; aprendizagem estratégia didática e trabalho social.

\section{Introducción}

En la actualidad los procesos de enseñanza, afirman Acosta y Boscán (2014), implementados por la mayoría de los docentes, se encuentran inmersos en modelos educativos tradicionales, con un enfoque conductista en la que no se planifican académicas de modo reflexivo y creativo. Esta posición, no permite el pleno desarrollo de las competencias del estudiante, privando la innovación pedagógica en los profesionales de la enseñanza, trayendo como consecuencias la apatía y desmotivación del estudiantado.

Es por ello que se deben utilizar estrategias de aprendizajes que pueden ser estimulantes para el desarrollo de las capacidades intelectuales y afectivas, como la que brinda el contacto con la naturaleza; sin embargo, los profesores cuando planifican sus unidades didácticas, en pocas o en ninguna ocasión sugieren los trabajos de campo, (Monereo, 2009). 
El trabajo de campo es una herramienta activa del proceso de aprendizaje, puesto que, durante su desarrollo, los educandos participan, experimentan, analizan, reflexionan, descubren hechos, desarrollan conceptos y perciben de manera real la interrelación del hombre con su medio. (Pérez, et al. 2014). Por lo tanto, es importante la realización de trabajo de campo ya que es una estrategia didáctica, que permite acercar al individuo con el entorno y conocer la diversidad de seres vivos que habitan en el ambiente; posibilitando un medio de aprendizaje mucho más dinámico y vivencial, rompiendo con la monotonía de un aula de clase, donde el estudiante es capaz de confrontar la teoría con lo práctica.

Cabe señalar, que desde el paradigma complejo el trabajo de campo permite promover una interpretación holística de la realidad. El abordaje científico, se hace en forma inter y transdisciplinar, lo que se traduce en un compromiso del hombre con la sociedad y el medio; en un intento por reconstruir una unidad de concepto que se haya perdido al fragmentarse los conocimientos. De allí que un planteamiento constructivista, propone enseñar a pensar y a actuar sobre contenidos significativos y contextuales; según Vera y Martínez (2013) una propuesta para construir conocimientos es la investigación dirigida, lo cual acerca al estudiante con el trabajo científico, siendo necesario valorar los conocimientos previos del estudiante, ya que constituyen sistemas de interpretación desde los cuales otorgan significado a las situaciones de aprendizaje.

Así mismo, es necesario que los profesores realicen actividades de campo que promuevan los procesos de observación, recolección de información, interpretación, formulación de hipótesis y experimentación, que incentive a los estudiantes a leer, pensar y reconstruir lo que observan en su entorno. De igual modo, desarrollan destrezas y habilidades psicomotoras que faciliten la solución de problemas de naturaleza práctica; además, fortalecen hábitos y valores que les permitan desenvolverse como individuo integrante de la sociedad. Para lograr lo anteriormente expuesto, es importante que los docentes dejen a un lado la monotonía educacional e implementen los trabajos de campo, debido a todos los beneficios que brinda; ofreciendo la posibilidad de aprender de una manera diferente.

Al respecto, indican Pérez y Rodríguez (2006), los trabajos o actividades de campo es una estrategia didáctica que acerca de manera consciente al individuo con la realidad, son una oportunidad de enseñanza y aprendizaje valioso para el maestro y el estudiante. Por su parte Vera 
y Martínez (2013), indican que la práctica de campo permite ganar valiosas experiencias que demandan la identificación, comprensión y el empleo de un vocabulario disciplinar que incluye términos, procesos y aspectos metodológicos, entre otros. Por tanto, dicha estrategia, rompe con la monotonía educativa, haciendo del proceso una experiencia vivencial, donde los aspectos procedimentales y actitudinales cobran valor, más allá de lo meramente teórico.

\section{Metodología}

E El estudio siguió una metodología de tipo descriptivo con un diseño de campo porque se realizó en el lugar en donde se dio el problema, y no experimental dado que no se manipularon las variables en estudio. La población estuvo constituida por 80 estudiantes de Trabajo social de la ULEAM, en la ciudad de Manta, durante el primer semestre del año 2017. La información se obtuvo a través de un instrumento tipo cuestionario y la técnica de aplicación fue la encuesta. La estadística aplicada fue de tipo descriptiva, cuyos resultados se presentaron en tablas con sus respectivos gráficos.

\section{Resultados}

El análisis de los resultados se presenta en tablas con sus perspectivas evaluaciones, los mismos se presenta en 5 tablas con sus respectivos gráficos.

Tabla 1. El Proceso de observación como una herramienta didáctica

\begin{tabular}{|c|c|c|}
\cline { 2 - 3 } \multicolumn{1}{c|}{} & \multicolumn{2}{c|}{ Estudiantes } \\
\hline CATEGORIAS & FR & $\%$ \\
\hline Alto Aprendizaje & 62 & 77 \\
\hline Medio Aprendizaje & 15 & 19 \\
\hline Bajo Aprendizaje & 3 & 4 \\
\hline
\end{tabular}

Fuente: Elaboración propia. 


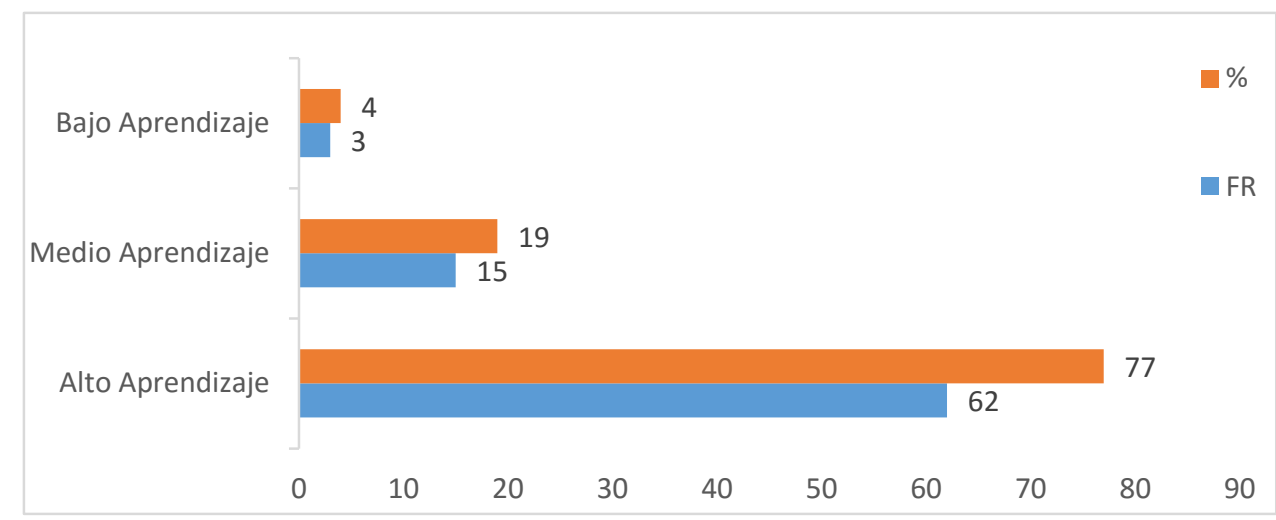

Grafico 1. El Proceso de observación como una herramienta didáctica

Fuente: Elaboración Propia

En la tabla y gráfico $\mathrm{N}^{\circ} 1$ se presenta el análisis descriptivo frecuencial del proceso de observación como una herramienta didáctica, en la que se indica que el $77 \%$ del total de los estudiantes evaluados indico un alto aprendizaje a través del proceso de observación como herramienta didáctica para el aprendizaje en la carrera de trabajo social, convirtiéndose en un modo primario de acercarse a su realidad y generar nuevos conocimientos, el cual se reproduce en el desarrollo del aprendizaje de cada estudiante, es una potencialidad que se trae y que poco a poco se va construyendo esa capacidad en el proceso de crecimiento en el camino del conocimiento. Seguido del $19 \%$ que se evaluaron en la categoría de aprendizaje medio y en menor porcentaje se indicó la categoría bajo aprendizaje con solo el $4 \%$ del total de las evaluaciones.

Tabla 2. El Proceso de Recolección de la información como una herramienta didáctica

\begin{tabular}{|c|c|c|}
\cline { 2 - 3 } \multicolumn{1}{c|}{} & \multicolumn{2}{c|}{ Estudiantes } \\
\hline CATEGORIAS & Fr & $\%$ \\
\hline Alto Aprendizaje & 65 & 81 \\
\hline Medio Aprendizaje & 14 & 17 \\
\hline Bajo Aprendizaje & 1 & 2 \\
\hline
\end{tabular}

Fuente: Elaboración Propia. 


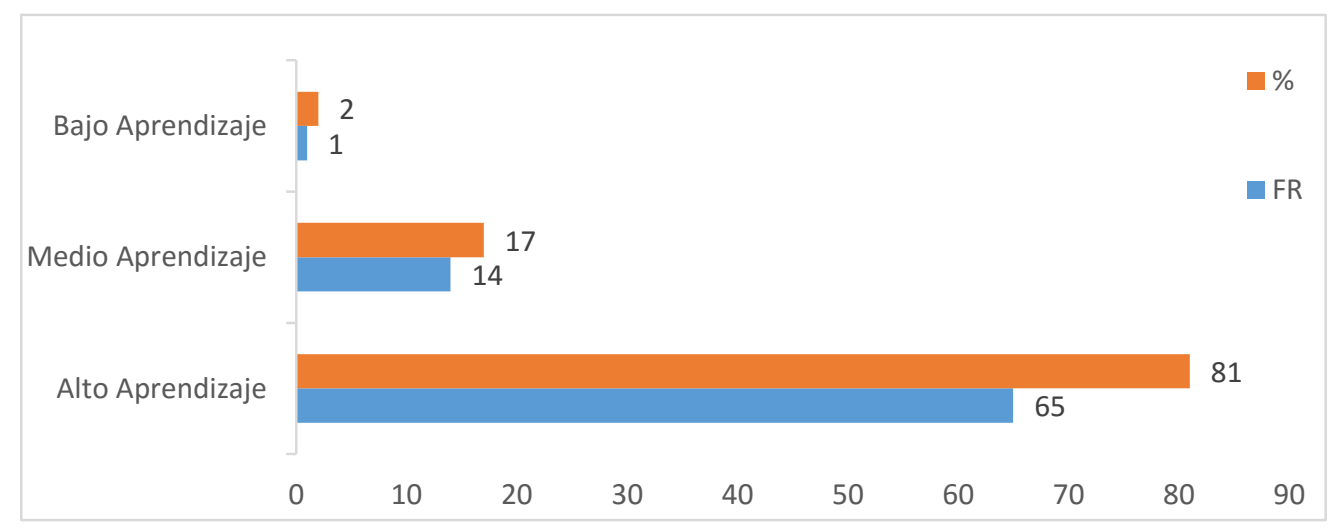

Grafico 2. El Proceso de Recolección de la información como una herramienta didáctica Fuente: Elaboración Propia

En la tabla y gráfico $\mathrm{N}^{\mathrm{o}} 2$ se presenta el análisis descriptivo frecuencial del proceso de Recolección de la información como una herramienta didáctica, en la que se indica que el $81 \%$ del total de los estudiantes evaluados indico un alto aprendizaje a través de la recolección de la información como herramienta didáctica para el aprendizaje en la carrera de trabajo social, durante este proceso de desarrolla la comprensión y entendimiento de las ideas que simbólicamente están siendo comunicados a través del texto. Seguido del 14\% que se evaluaron en la categoría de aprendizaje medio y en menor porcentaje se indicó la categoría bajo aprendizaje con solo el $2 \%$ del total de las evaluaciones.

Tabla 3. El Proceso de interpretación de la información como una herramienta didáctica.

\begin{tabular}{|c|c|c|}
\cline { 2 - 3 } \multicolumn{1}{c|}{} & \multicolumn{2}{c|}{ Estudiantes } \\
\hline CATEGORIAS & Fr & 54 \\
\hline Alto Aprendizaje & 43 & 40 \\
\hline Medio Aprendizaje & 32 & 6 \\
\hline Bajo Aprendizaje & 5 & \\
\hline
\end{tabular}

Fuente: Elaboración Propia. 


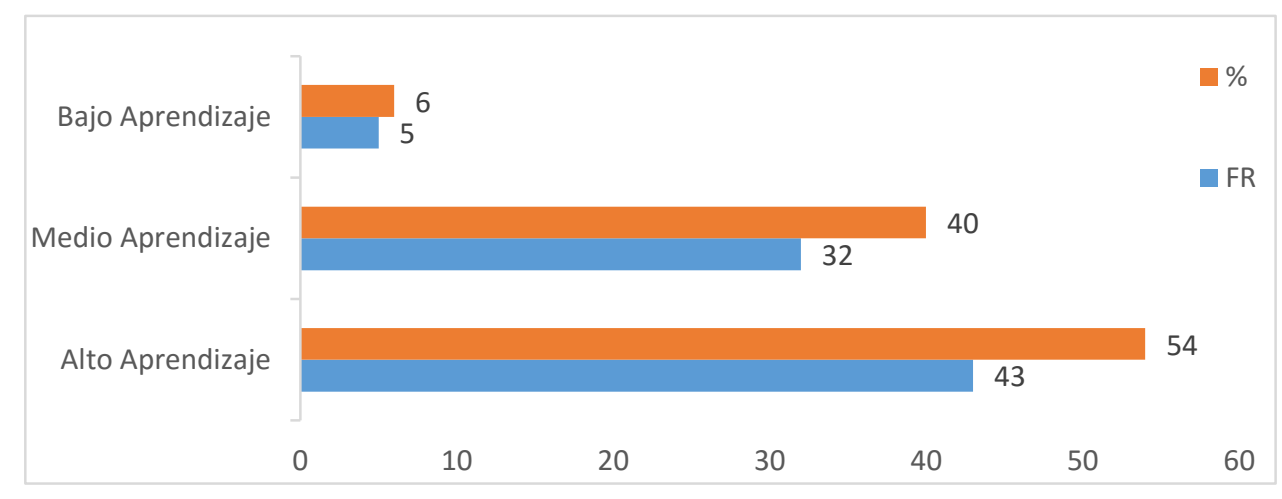

Grafico 3. El Proceso de interpretación de la información como una herramienta didáctica.

Fuente: Elaboración Propia

En la tabla y gráfico $\mathrm{N}^{\mathrm{o}} 3$ se presenta el análisis descriptivo frecuencial del proceso de interpretación de la información como una herramienta didáctica, en la que se observa que el $54 \%$ del total de los estudiantes evaluados indico un alto aprendizaje a través del proceso de interpretación de la información como herramienta didáctica para el aprendizaje en la carrera de trabajo social, en la cual los alumnos aprenden a través de un proceso donde los alumnos aprenden a pensar y a participar activa, reflexiva y creadoramente. Seguido del $40 \%$ que se evaluaron en la categoría de aprendizaje medio y en menor porcentaje se indicó la categoría bajo aprendizaje con solo el $6 \%$ del total de las evaluaciones.

Tabla 4. El Proceso de Formulación de hipótesis como una herramienta didáctica.

\begin{tabular}{|c|c|c|}
\cline { 2 - 3 } \multicolumn{1}{c|}{} & \multicolumn{2}{c|}{ Estudiantes } \\
\hline CATEGORIAS & Fr & $\%$ \\
\hline Alto Aprendizaje & 32 & 40 \\
\hline Medio Aprendizaje & 38 & 47 \\
\hline Bajo Aprendizaje & 10 & 13 \\
\hline
\end{tabular}

Fuente: Elaboración Propia. 


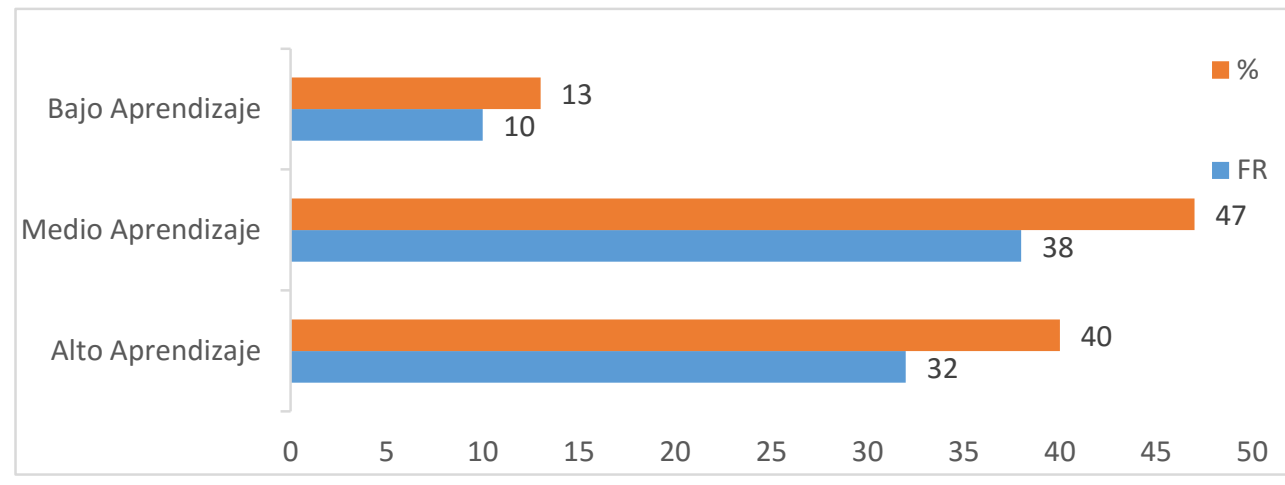

Grafico 4. El Proceso de Formulación de hipótesis como una herramienta didáctica

Fuente: Elaboración Propia

En la tabla y gráfico $\mathrm{N}^{\circ} 4$ se presenta el análisis descriptivo frecuencial del proceso de Formulación de hipótesis como una herramienta didáctica, en la que se indica que el $47 \%$ del total de los estudiantes evaluados indico un aprendizaje medio a través del proceso de Formulación de hipótesis como herramienta didáctica para el aprendizaje en la carrera de trabajo social, que permite resolver en cierta medida el problema de dar sentido al que enseña y al proceso de construcción del conocimiento. Asimismo, se trata de una herramienta didáctica útil para el proceso de enseñanza aprendizaje que permita orientar la exploración y comprensión de las dificultades que los alumnos encuentran en el proceso de construcción de conocimientos. Seguido del $40 \%$ que se evaluaron en la categoría de alto aprendizaje y en menor porcentaje se indicó la categoría bajo aprendizaje con solo el 13\% del total de las evaluaciones.

Tabla 5. El Proceso de la experimentación como una herramienta didáctica.

\begin{tabular}{|c|c|c|}
\cline { 2 - 3 } \multicolumn{1}{c|}{} & \multicolumn{2}{c|}{ Estudiantes } \\
\hline CATEGORIAS & Fr & $\%$ \\
\hline Alto Aprendizaje & 41 & 51 \\
\hline Medio Aprendizaje & 28 & 35 \\
\hline Bajo Aprendizaje & 11 & 14 \\
\hline
\end{tabular}

Fuente: Elaboración Propia. 


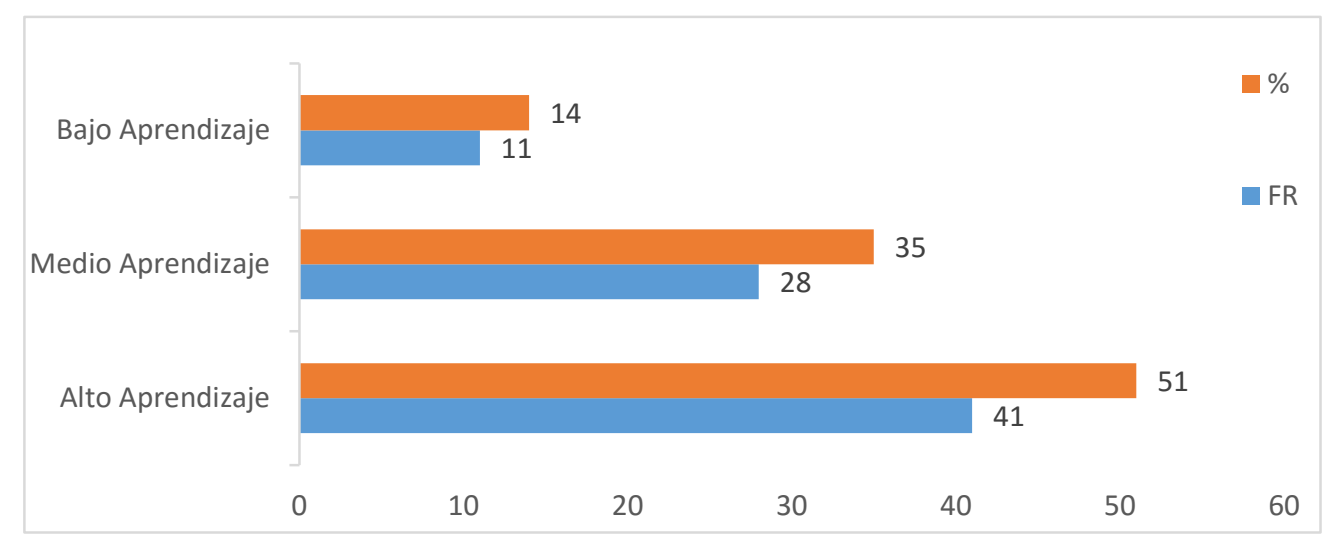

Grafico 5. El Proceso de la experimentación como una herramienta didáctica

Fuente: Elaboración Propia

En la tabla y gráfico $\mathrm{N}^{\circ} 5$ se presenta el análisis descriptivo frecuencial del proceso de experimentación como una herramienta didáctica, en la que se indica que el $51 \%$ del total de los estudiantes evaluados revelo un alto aprendizaje a través del proceso de experimentación como herramienta didáctica para el aprendizaje en la carrera de trabajo social. Seguido del 35\% que se evaluaron en la categoría de aprendizaje medio y en menor porcentaje se indicó la categoría bajo aprendizaje con solo el 14\% del total de las evaluaciones. El uso de la experimentación, como herramienta metodológica, la explicación y la aplicación de los denominados organizadores previos, empleados como conectores de índole cognitivo entre los pre saberes del educando y la nueva información que el docente lleva al momento de impartir sus conocimientos.

\section{Conclusiones}

Existe un alto índice de aprendizaje a través del proceso de observación como herramienta didáctica, convirtiéndose en un modo primario de acercarse a su realidad y generar nuevos conocimientos.

La recolección de la información como una herramienta didáctica, es concebida como de alto aprendizaje en la carrera de trabajo social, en la que es posible el desarrollo de la comprensión y entendimiento de las ideas que simbólicamente están siendo comunicados a través del texto.

Se indicó un alto aprendizaje a través del proceso de interpretación de la información como una herramienta didáctica, en la cual los alumnos aprenden a través de un proceso donde los alumnos logran pensar, participar activamente, reflexionar y ser creadores. 
El proceso de formulación de hipótesis como una herramienta didáctica, se evaluó como un aprendizaje medio en cuanto al sentido de lo que se enseña y el proceso de construcción del conocimiento por parte de los alumnos.

El proceso de experimentación como una herramienta didáctica, fue considerada de alto aprendizaje en la carrera de trabajo social, donde la experimentación desde lo metodológico, su explicación y aplicación entre los pre - saberes del educando y la nueva información permite que el docente logre impartir sus conocimientos.

\section{Referencias Bibliográficas}

Acosta S y Finol. (2015). Competencias de los docentes de Biología en las universidades públicas. Revista de Estudios Interdisciplinarios en Ciencias Sociales. (Universidad Rafael Belloso Chacín). Vol. 17, (2). Maracaibo-Venezuela. pp. 208-224.

Monereo C. (2009). Profesores y alumnos estratégicos. Cuando aprender es consecuencia de pensar. Madrid-España: Editorial Pascal.

Pérez A y Rodríguez L. (2006). La salida de campo: una manera de enseñar y aprender Geografía. Geoenseñanza. Vol.11- (2). pp. 229-234.

Pérez R. (2014). El trabajo de campo, una estrategia para la enseñanza de la Zoología. IV Jornadas Nacionales de investigación estudiantil, de la Universidad del Zulia (Redieluz). Maracaibo- Venezuela

Vera A y Martínez M. (2013). Bosque de manglar: ambiente para la enseñanza y aprendizaje de la Ecología. Multiciencias. Vol. 13 (1). pp. 46-52. 